\title{
Cost-effectiveness of phosphate binders among patients with chronic kidney disease not yet on dialysis: a long way to go
}

Rana Rizk@

\begin{abstract}
Hyperphosphatemia management is integral to the management of patients with chronic kidney disease. This mineral abnormality is associated with greater costs, but so is its management, especially with the use novel phosphate binders. The economic evaluation of these pharmaceutical agents is increasingly needed to provide evidence for value of money spent and inform resource allocation. Recently, Nguyen et al. explored the economical attractiveness of Sevelamer relative to Calcium Carbonate among patients with chronic kidney disease not yet on dialysis and concluded that the former was cost-effective. The current commentary discusses the results of this analysis and sheds light on the methodological challenges of economic evaluations in this field.
\end{abstract}

Keywords: Chronic kidney disease, Phosphate binders, Hyperphosphatemia, Cost-effectiveness analysis

\section{Background}

Hyperphosphatemia is consistently and independently associated with increased morbidity and mortality among end stage renal disease patients [1-3], and results in financial burdens for health systems [4]. Although this issue was explored to a lesser extent among patients with chronic kidney disease (CKD), results from large studies suggest an independent association between elevated serum phosphorus levels and increased patient mortality risk $[5,6]$. The association between hyperphosphatemia and progression to dialysis initiation has also been suggested. However, this association was inconsistent across studies [7] and was rendered not statistically significant when potential confounders were accounted for in the analysis [6]. Despite this, serum phosphorus management in CKD patients has gained increasing importance in contemporary nephrology practice, and tight targets for serum phosphorus levels were set for these patients, although, up-till-now, we still lack conclusive evidence, ie prospective interventional studies, to

Correspondence: r.rizk@maastrichtuniversity.nl

Department of Health Services Research, CAPHRI School of Public Health and Primary Care, Maastricht University, 6200 MD Maastricht, The Netherlands demonstrate that reduction in serum phosphate improves CKD patient outcomes.

Concerns about the high cost of hyperphosphatemia's consequences are offset by concerns about hyperphosphatemia's high treatment cost [8], especially with the novel pharmaceuticals, ie non-calcium-based phosphate binders. Within this scope, economic evaluations -studies providing evidence for value of money spent- are now being increasingly used by public health decision makers to guide the allocation of scarce resources [9].

\section{Main text}

The cost-effectiveness of phosphate binders was first addressed among hemodialysis patients, where due to many factors, the management of serum phosphorus is a continuous challenge. Since 2005, multiple full economic evaluations were published in this regards. In 2016, the comparative cost-effectiveness of these agents was systematically explored by Rizk et al. [10], where in view of the suboptimal quality and inconsistent results of included studies, robust conclusions could not be generated. The authors of the review suggested that calciumbased binders- especially Calcium Acetate was the most economically attractive therapy, in first-line and 
sequential use, in prevalent patients, and that Lanthanum Carbonate might provide good value for money, as second-line therapy, in incident patients. In that review, the incremental cost-utility ratio of Sevelamer relative to Calcium Carbonate ranged between US $\$ 36,803$ and US\$157,760 per quality adjusted life year (QALY) gained for first-line use among prevalent patients. Relative to the same comparator, Sevelamer was borderline cost-effective among incident patients (US\$47,153).

So far, the comparative cost-utility of phosphate binders among renal patients not yet on dialysis was only explored in two studies. The first by Vegter et al. [11] concluded that second-line Lanthanum Carbonate dominated -less costly and more effective-calcium-based binders. Cost savings were mainly attributed to the delayed CKD progression and dialysis initiation. The second study by Thompson et al. [12] concluded that firstline Sevelamer was cost-effective compared with Calcium Carbonate (incremental cost of $£ 23,878$ per QALY gained (US\$36,475)). However, in this study, the results were most sensitive to alternative assumptions regarding the impact of Sevelamer on dialysis initiation. Both studies $[11,12]$ adopted the perspective of the National Health Service in the United Kingdom and used a lifelong Markov model. Accordingly, one would suggest that the cost-effectiveness of non-calcium based binders is mainly driven by their perceived effect on delaying the treatment by dialysis.

This issue of BMC Nephrology includes the third full economic evaluation of phosphate binders among patients with CKD not yet on dialysis. The article by Nguyen et al. [13] assessed the life-time incremental cost-utility of first-line Sevelamer relative to Calcium Carbonate from the perspective of a third party payer in Singapore, using a Markov model. This study was funded by Sanofi-Aventis (Singapore). The authors concluded that Sevelamer produces an incremental costutility ratio of $S \$ 51,756$ (US\$38,500) per QALY gained relative to Calcium Carbonate.

In contrast to the above-mentioned two studies, where country-specific clinical evidence was readily available for the analysis, Nguyen et al. [13] employed a multitude of clinical effectiveness sources to construct their model, some of which were extracted from divergent patient population groups. In order to circumvent limitations of available Singapore-specific data, especially survival outcomes (mortality risks among Singaporean CKD and dialysis patients), the authors used age-specific mortality risks from Singapore life-tables, mortality hazard ratios for CKD patients based on a large Taiwanese cohort study and adjusted age-specific mortality risks from the United States Renal Data System. However, other key parameters used in the model, such as the probabilities for transitioning to dialysis or the needed dose of
Sevelamer to reach a certain level of effectiveness were directly extrapolated from divergent patient groups [14] to the study's population. This issue must be revisited and further research in this regards must be conducted in order to inform public health decision making, especially that the sensitivity analyses showed that Sevelamer became less economically attractive with higher dialysis costs and at higher Sevelamer doses. Finding readily available country-specific data for model inputs or information to adjust data from other countries is a consistent challenge across model-based economic evaluation studies, especially those conducted in low and middle income countries. This was repeatedly faced by authors from these countries in the field of phosphate-binder related research among renal patients [10].

Similarly, although Nguyen et al. [13] presented thorough details of the data used for constructing the model, caution must be taken when directly extrapolating their results to be used in other countries. In fact, the incremental cost-utility ratio of Sevelamer versus Calcium Carbonate was most sensitive to changes in the cost of the former. This agent was shown to be no longer costeffective at the price of $\$ \$ 1.69 / \mathrm{g}$ (US $\$ 1.26 / \mathrm{g}$ ). Given the changes in the costs of therapeutic agents across countries $[15,16]$, the direct transferability of these results is questioned [16]. This issue was also pinpointed in other areas of economic evaluations of health care interventions [17]. In fact, strong evidence points out the challenging and complex task of the transferability of economic evaluation data across countries, and that transferability requires a minimum of country-specific substitution of practice pattern data, in addition to unit cost data [16].

On the other hand, Grima et al. [18] presented the case for excluding dialysis costs in economic evaluations of interventions that increase survival, such as phosphate binders. The authors argued that due to the high cost of dialysis, the inclusion of its costs in the analysis of any life-extending intervention eliminates the possibility of obtaining a favorable cost-effectiveness ratio, regardless of the clinical benefits of the intervention. According to the authors, this might deny renal patients access to interventions that are initially cost-effective. Assessing the impact of excluding future dialysis costs in the sensitivity analyses would have presented a more realistic look at the cost-effectiveness of Sevelamer. Moreover, noncalcium based phosphate binders seem to be more economically attractive when used as a second line therapy among dialysis and non-dialysis patients [10, 11]. In depth exploration of this issue would have been interesting in order to inform decision making, especially in countries with limited health resources.

This study was funded by a pharmaceutical company. Previous systematic reviews of the literature [19, 20] 
found that pharmaco-economic studies sponsored by the industry were more likely to favor the sponsor's product. This was also the case of publications on phosphate binders for hyperphosphatemia management [10]. Sponsor bias (specifically in trial-based economic evaluations) was thoroughly discussed by Evers et al. [21] and solutions to overcome it, beyond disclosure of the financial conflict of interest and a rigorous peer-review process, included maintenance of good methodological standards. This highlights the pressing need for stringent guidelines governing the conduct and publication of economic evaluations.

Finally, in contrast to the case of hemodialysis patients, where various evidence-based practice guidelines agree on actively managing hyperphosphatemia, this issue is less clear among patients not receiving dialysis. While the Kidney Disease: Improving Global Outcomes (KDIGO) guidelines [22] recommend maintaining serum phosphate levels "within the normal range" starting stage 3 or 4 of the disease, the United Kingdom National Institute for Health and Clinical Excellence (NICE) guidelines [23] suggest that serum phosphate should be monitored routinely only in stages 4,5 , and $5 \mathrm{~d}$. Nevertheless, in all of the above-mentioned cases, the guidelines were not based on definitive evidence on the beneficial effect of lowering phosphate levels in early or late CKD.

\section{Conclusion}

In conclusion, evaluating the outcomes of the implementation of renal guidelines pertaining to serum phosphorus management in terms of reduced morbidity, mortality and delayed dialysis initiation is becoming imperative. This would pave the way for a more rationale evaluation of the cost-effectiveness of interventions in this regards. Until then, employing borderline costeffective phosphate binders to achieve tight serum phosphorus levels, where supportive conclusive evidence surrounding the clinical or financial benefits is lacking, is to be discussed in the light of the country-specific health priorities and budgetary limitations.

\section{Abbreviations}

CKD, chronic kidney disease; KDIGO, kidney disease: improving global outcomes; NICE, National Institute for Health and Clinical Excellence; QALY, quality adjusted life year

\section{Acknowledgements}

None.

\section{Funding}

None.

\section{Availability of data and materials}

All information supporting the conclusions of this manuscript are included within the article. Any additional information can be obtained upon request.

\section{Competing interests}

The author declares that she has no competing interests.

Consent for publication

Not applicable.

Ethical approval and consent to participate

Not applicable.

Received: 21 June 2016 Accepted: 24 June 2016

Published online: 08 July 2016

References

1. Block GA, Hulbert-Shearon TE, Levin NW, Port FK. Association of serum phosphorus and calcium $\mathrm{x}$ phosphate product with mortality risk in chronic hemodialysis patients: a national study. Am J Kidney Dis. 1998;31:607-17.

2. Young EW, Akiba T, Albert JM, et al. Magnitude and impact of abnormal mineral metabolism in hemodialysis patients in the Dialysis Outcomes and Practice Patterns Study (DOPPS). Am J Kidney Dis. 2004:44:34-8.

3. Tentori F, Blayney MJ, Albert JM, et al. Mortality risk for dialysis patients with different levels of serum calcium, phosphorus, and PTH: The Dialysis Outcomes and Practice Patterns Study (DOPPS). Am J Kidney Dis. 2008;52: 519-30.

4. White DA, Odedina F, Xiao H, Campbell E, Segal R. The economic burden of end-stage renal disease with hyperphosphatemia. Dis Manag Health Outcomes. 2012;14:99-106.

5. Kestenbaum B. Serum Phosphate levels and mortality risk among people with chronic kidney disease. J Am Soc Nephrol. 2005;16:520-8.

6. Selamet $\mathrm{U}$, Tighiouart $\mathrm{H}$, Sarnak MJ, et al. Relationship of dietary phosphate intake with risk of end-stage renal disease and mortality in chronic kidney disease stages 3-5: The Modification of Diet in Renal Disease Study. Kidney Int. 2016:89:176-84.

7. Mehrotra R, Peralta CA, Chen S-C, et al. No independent association of serum phosphorus with risk for death or progression to end-stage renal disease in a large screen for chronic kidney disease. Kidney Int. 2013;84:989-97.

8. White CA, Jaffey J, Magner P. Cost of applying the K/DOQI guidelines for bone metabolism and disease to a cohort of chronic hemodialysis patients. Kidney Int. 2007;71:312-7.

9. Brousselle A, Lessard C. Economic evaluation to inform health care decision-making: Promise, pitfalls and a proposal for an alternative path. Soc Sci Med. 2011;72:832-9.

10. Rizk R, Hiligsmann M, Karavetian M, Evers SM. Economic evaluations of interventions to manage hyperphosphataemia in adult haemodialysis patients: A systematic review. Nephrology. 2016;21:178-87.

11. Vegter S, Tolley K, Keith MS, Postma MJ. Cost-effectiveness of lanthanum carbonate in the treatment of hyperphosphatemia in chronic kidney disease before and during dialysis. Value Health. 2011:14:852-8.

12. Thompson M, Bartko-Winters S, Bernard L, Fenton A, Hutchison C, Di lorio B. Economic evaluation of sevelamer for the treatment of hyperphosphatemia in chronic kidney disease patients not on dialysis in the United Kingdom. J Med Econ. 2013;16:744-55.

13. Nguyen HV, Bose S, Finkelstein E. Incremental cost-utility of sevelamer relative to calcium carbonate for treatment of hyperphosphatemia among pre-dialysis chronic kidney disease patients. BMC Nephrol. 2016;17:45.

14. Di lorio B, Bellasi A, Russo D, on behalf of the INDEPENDENT Study Investigators. Mortality in kidney disease patients treated with phosphate binders: A randomized study. Clin J Am Soc Nephrol. 2012;7:487-93.

15. Drummond M, Barbieri M, Cook J, et al. Transferability of economic evaluations across jurisdictions: ISPOR Good Research Practices Task Force report. Value Health. 2009;12:409-18.

16. Goeree R, Burke N, O'Reilly D, Manca A, Blackhouse G, Tarride JE. Transferability of economic evaluations: approaches and factors to consider when using results from one geographic area for another. Curr Med Res Opin. 2007:23:671-82.

17. Elliott RA, Putman K, Davies J, Annemans L. A review of the methodological challenges in assessing the cost effectiveness of pharmacist interventions. PharmacoEconomics. 2014:32:1185-99.

18. Grima DT, Bernard MLM, Dunn ES, McFarlane PA, Mendelssohn DC. Cost-effectiveness analysis of therapies for chronic kidney disease patients on dialysis. PharmacoEconomics. 2012;30:981-9. 
19. John-Baptiste A, Bell C. Industry sponsored bias in cost effectiveness analyses. BMJ. 2010;341:c5350.

20. Lexchin J, Bero LA, Djulbegovic B, Clark O. Pharmaceutical industry sponsorship and research outcome and quality: Systematic review. BMJ. 2003;326:1167-70

21. Evers SMAA, Hiligsmann M, Adarkwah CC. Risk of bias in trial-based economic evaluations: Identification of sources and bias-reducing strategies. Psychol Health. 2015;30:52-71.

22. Kidney Disease: Improving Global Outcomes (KDIGO) CKD-MBD Work Group. KDIGO clinical practice guideline for the diagnosis, evaluation, prevention, and treatment of chronic kidney disease-mineral and bone disorder (CKD-MBD). Kidney Int. 2009;76 Suppl 113:1-130.

23. Dasgupta I, Shroff R, Bennett-Jones D, McVeigh G. Management of hyperphosphataemia in chronic kidney disease: Summary of National Institute for Health and Clinical Excellence (NICE) guideline. Nephron Clin Pract. 2013;124:1-9.

Submit your next manuscript to BioMed Central and we will help you at every step:

- We accept pre-submission inquiries

- Our selector tool helps you to find the most relevant journal

- We provide round the clock customer support

- Convenient online submission

- Thorough peer review

- Inclusion in PubMed and all major indexing services

- Maximum visibility for your research

Submit your manuscript at www.biomedcentral.com/submit

\section{) Biomed Central}

